Neuer Hausarzt-EBM

\title{
Wann wird die Grundpauschale reduziert?
}

\author{
In MMW Nr. 2/2013, S. 8 stellten wir \\ den Entwurf eines neuen Hausarzt- \\ EBMs vor. Dazu ein Leser:
}

— Die einschlägigen Medien und ihre Autoren, wie wiederholt auch G. Zimmermann, betonen im Zusammenhang mit dem geplanten neuen EBM, dass bei gleichzeitiger Erbringung von Leistungen wie Akupunktur, Psychotherapie und "spezielle" Diabetologie die Grundpauschale geteilt und der Chronikerzuschlag wegfallen soll. Es wäre zu klären und öffentlich darzustellen, ob sich diese Vorgehensweise auf das gesamte Klientel einer Praxis bezieht oder nur bei Überschreitung eines bestimmten Anteils dieser Leistungen am gesamten Leistungsaufkommen oder nur beim einzelnen Patienten, welcher die genannten Leistungen auslöst.

- Dr. Günther Schäfer

\section{Antwort von Dr. G. Zimmermann:}

Der Entwurf zum neuen Hausarzt-EBM, der im Moment auf KBV-Ebene diskutiert wird, sieht tatsächlich eine Regelung nach dem Prinzip des bisherigen sog. K.O.-Kataloges vor. Das bedeutet, eine einzige Leistung aus dem vorgesehenen Katalog für „atypische Hausarztleistungen" reicht aus, um nur noch in den "Genuss" der reduzierten Pauschale zu kommen. Das bezieht sich dann allerdings nur auf diesen Fall.

\section{Ginseng}

\section{Hilft der wirklich?}

Frage von N. N.:

Kürzlich wurde in einer Talkshow im Fernsehen über Ginseng und seine (für mich eher fraglichen) Effekte gesprochen. Und schon wurde ich auf einer Party als Arzt hierzu befragt, konnte aber, da Chirurg, eher keine fachlich fundierte Antwort geben. Was ist denn nun daran? Wenn es hilft, hole ich es mir auch sofort aus der nächsten Apotheke!

Antwort von A. Rolfes-Bußmann und J. C. Frölich:

In der Monographie zur Ginsengwurzel gibt die Kommission E des BfArM folgendes Anwendungsgebiet an: „als Tonikum zur Stärkung und Kräftigung bei Müdigkeits- und Schwächegefühl, nachlassender Leistungs- und Konzentrationsfähigkeit sowie in der Rekonvaleszenz".
Zuverlässige Studiendaten zur klinischen Wirksamkeit von Ginseng beim Menschen fehlen. Eine schädliche Wirkung ist bei bestimmungsgemäßer Anwendung jedoch nicht zu erwarten.

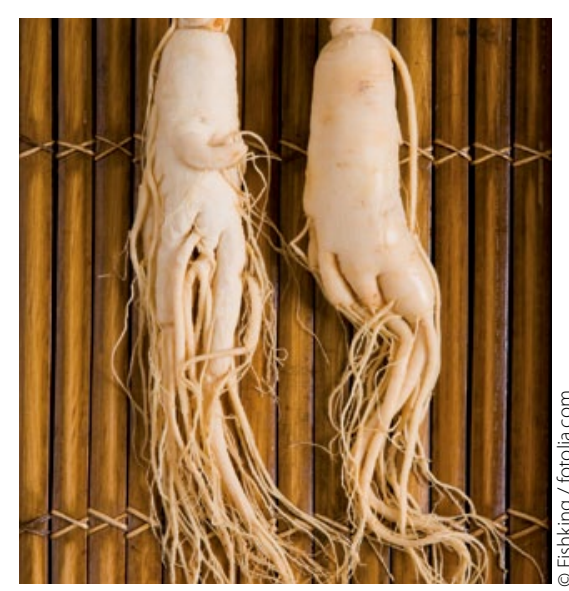

Ginsengwurzel: In Asien ein Sinnbild für Gesundheit und langes Leben.
Hier ist aber noch nicht das ,letzte Wort" gesprochen. Hausärzteverband und Internistenverband haben ihren energischen Widerstand gegen diese Form der EBM-Reform angekündigt. Ich persönlich rechne deshalb auch nicht mit einer Umsetzung zum 1.7.2013. Wenn überhaupt wird es zu einer einheitlichen EBM-Reform im haus- und fachärztlichen Bereich kommen. Hier hat die KBV einen Zeitrahmen bis 2015 vorgegeben.

- Dr. Gerd W. Zimmermann, Facharzt für Allgemeinmedizin, Kapellenstraße 9, D-65719 Hofheim

Dennoch sollte man berücksichtigen, dass auch bei Mitteln mit unzureichend belegter Wirksamkeit möglicherweise Wechselwirkungen mit anderen Arzneimitteln auftreten können. So werden für Ginseng Interaktionen mit Digoxin (erhöhte Digoxin-Serumkonzentrationen), MAO-Inhibitoren (erhöhte Toxizität der MAO-Hemmer), Warfarin (reduzierte Wirkung von Warfarin) und Imatinib (erhöhte Lebertoxizität von Imatinib) erwartet.

- Dr. A. Rolfes-Bußmann, Apothekerin, Prof. Dr. med. J. C. Frölich, Institut für Klinische Pharmakologie, Medizinische Hochschule Hannover, D-30623 Hannover 